\title{
Ischemic Acute Renal Failure and Antioxidant Therapy in the Rat The Relation between Glomerular and Tubular Dysfunction
}

\author{
J. E. Bird," K. Milhoan," C. B. Wilson, ${ }^{\star}$ S. G. Young," C. A. Mundy, \\ *Department of Medicine, University of California, San Diego School of Medicine, and Veterans Administration Hospital, San Diego, \\ California 92161; ' Department of Immunopathology, Research Institute of Scripps Clinic, La Jolla, California 92037
}

\begin{abstract}
The effects of antioxidant therapy with probucol were evaluated in rats subjected to $1 \mathrm{~h}$ renal ischemia and to $24 \mathrm{~h}$ reperfusion. Probucol exerted significant antioxidant effects in renal cortical tubules in vitro when exposed to a catalase-resistant oxidant. At 24 h probucol treatment (IP) improved single nephron glomerular filtration rate (SNGFR) $(28.1 \pm 3.3 \mathrm{nl} /$ $\mathrm{min}$ ) in comparison to untreated ischemic (I) rats (15.2 \pm 3.0$)$, primarily as a result of improving SNGFR in a population of low SNGFR, low flow and/or obstructed nephrons. However, absolute proximal reabsorption remained abnormally low in IP rats at $24 \mathrm{~h}(5.9 \pm 0.8 \mathrm{nl} / \mathrm{min})$, and cell necrosis was greater than in I rats. Kidney GFR remained low in IP rats due to extensive tubular backleak of inulin measured by microinjection studies. Evaluations after $2 \mathrm{~h}$ of reperfusion revealed a higher SNGFR in IP $(36 \pm 3.1 \mathrm{nl} / \mathrm{min})$ than I rats $(20.8 \pm 2.7$ $\hat{\mathbf{n}} \mathbf{l} / \mathbf{m i n})$. Absolute proximal reabsorption was essentially normal (11.6 $\pm 1.3 \mathrm{nl} / \mathrm{min})$ in IP rats, which was higher than IP rats at $24 \mathrm{~h}$ and the concurrent $I$ rats. Administration of the lipophilic antioxidant, probucol, increased SNGFR and proximal tubular reabsorption within $2 \mathrm{~h}$ after ischemic renal failure. Although SNGFR remained higher than I rats at $24 \mathrm{~h}$, absolute reabsorption fell below normal levels and tubular necrosis was more extensive in IP rats. Early improvement in nephron filtration with antioxidants may increase load dependent metabolic demand upon tubules and increase the extent of damage and transport dysfunction.
\end{abstract}

\section{Introduction}

Ischemic acute renal failure is a highly complex syndrome involving renal vasoconstriction, filtration failure, tubular obstruction, and tubular backleak of solutes (1). Because of this complexity, the measurement of one parameter of renal function such as total GFR to assess the benefit of a protective

Address reprint requests to Dr. Blantz, Veterans Administration Medical Center, 3350 La Jolla Village Drive, San Diego, CA 92161.

Presented in part in abstract form at a meeting of the American Society of Nephrology, 1986, the Western Society for Clinical Investigation, 1987, and the Federation of American Societies for Experimental Biology, 1987.

Received for publication 16 June 1987 and in revised form 30 October 1987.

1. Abbreviations used in this paper: APR, absolute proximal reabsorption; FR, fractional reabsorption; I, untreated ischemic; IP, probucol treated ischemic; IRF, ischemic renal failure; KHS, Krebs-Henseleit solution; MDA, malondialdehyde; MPO, myeloperoxidase; PAS, periodic acid Schiff; $P_{\mathrm{T}}$, tubular pressure; SNGFR, single nephron glomerular filtration rate; TBH, tert-butyl hydroperoxide.

The Journal of Clinical Investigation, Inc.

Volume 81, May 1988, 1630-1638 treatment may not be totally adequate. The contributing factors to ischemic renal failure (IRF) ${ }^{1}$ are multiple and time dependent events, any one of which may be separately affected by a protective approach.

Paller has reported that administration of oxygen radical scavengers provided some amelioration of ischemic damage in renal tissue (2). We have examined the protective effects of probucol, an antioxidant, in a rat model of ischemic renal failure. Probucol is a highly lipophilic drug that is approved for use in humans as a hypocholesterolemic agent (3). Its structure is diagrammed in Fig. 1. Probucol may inhibit atherogenesis by preventing the oxidative modification of low density lipoproteins by macrophages (4). Because of its lipophilic nature and small molecular size, probucol should insert into membranes in significant molar quantities and prevent lipid peroxidation induced by oxygen free radicals. The effects of probucol on renal structure and function were examined in rats after $1 \mathrm{~h}$ of renal ischemia. Evaluation of the time dependent effects of antioxidants was performed by comparison of glomerular and tubular function after 2 and $24 \mathrm{~h}$ reflow, allowing more complete characterization of the progression and pathogenesis of ischemic renal failure.

\section{Methods}

Experiments were performed on male Munich-Wistar rats (200-260 g). There were three treatment groups examined in this study: $(i)$ control (C) animals that received vehicle, and underwent unilateral nephrectomy and sham renal artery clamping; (ii) ischemic (I) animals that received vehicle, were unilaterally nephrectomized, and underwent left renal artery clamping for $60 \mathrm{~min}$; and (iii) ischemic probucol-treated (IP) animals that were treated with a $1 \%$ probucol diet or were injected with probucol $(300 \mathrm{mg} / \mathrm{kg}$ per d s.c.) for 2 wk before unilateral nephrectomy and left renal artery clamping. Probucol was a gift from Merrill Dow Research Institute (Cincinnati, Ohio). All rats were permitted free access to water. After 2 wk of treatment with vehicle or probucol, rats were anesthetized with $\mathrm{Na}$ pentobarbital (50 $\mathrm{mg} / \mathrm{kg}$ i.p.), a ventral midline incision was made, the right renal artery securely ligated, and the right kidney removed for analysis of tissue probucol levels using the HPLC method described by Satonin and Coutant (5). The left renal pedicle was dissected to expose the renal artery, and the artery was clamped for $60 \mathrm{~min}$ in the ischemic and probucol treated ischemic groups. The incisions were closed and the animals allowed to recover. Two groups of ischemic and probucol treated ischemic rats were maintained in cages for $7 \mathrm{~d}$ after surgery, with blood samples obtained from the tail vein for determination of serum creatinine levels on day $0,1,3,5$, and 7 .

\section{Micropuncture studies}

Rats were anesthetized with Inactin (100 mg/kg i.p.) 2 and $24 \mathrm{~h}$ after the ischemic period to perform micropuncture studies. Surgical preparation for micropuncture was as previously described (6). A tracheostomy was performed (PE-240) and PE-50 catheters were placed in the 


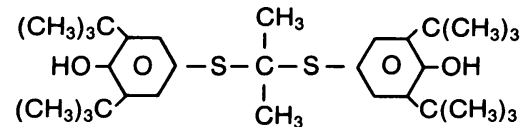

Figure 1. The chemical structure of the lipophilic antioxidant, probucol.

jugular vein, femoral artery, and left ureter. Arterial blood pressure was monitored with a pressure transducer and recorded on a Statham Instruments (Oxnard, CA), chart recorder. Body temperature was regulated on a heated table with a servocontrolled heating unit. Before micropuncture, the rats were infused with $1.0 \%$ body wt donor plasma for $60 \mathrm{~min}$ and $\left[{ }^{3} \mathrm{H}\right]$ inulin at a rate of $120 \mu \mathrm{Ci} / \mathrm{h}$. Donor plasma was then infused at $0.15 \%$ body $w t / h$.

Measurements of nephron filtration rate (SNGFR) were performed with the full recognition that models of IRF may exhibit considerable degrees of heterogeneity of filtration rate among nephrons. Late proximal tubules were localized in a highly random fashion for micropuncture by sampling tubules from the four quadrants of the micropuncture surface consecutively. In addition, the localizing pipette was positioned above the kidney surface, without viewing the surface concurrently, and the tubule immediately below this pipette was selected for evaluation. The proximal segments were localized by injection of FD\&DC green dye into the proximal tubule using 3-5- $\mu \mathrm{m}$ beveled-tip pipettes. Even if the injected dye did not move or moved slowly, suggesting low to negligible tubular flow rates, the tubule was injected with mineral oil and a 3-min collection was performed. The sample of tubular fluid was counted and if no aqueous fluid was collected, the value for SNGFR was considered to be zero. Tubular pressure $\left(P_{\mathrm{T}}\right)$ was monitored before and during tubular fluid collection. Sufficient counterpressure was applied to maintain $P_{\mathrm{T}}$ equal to precollection free flow pressure throughout the collection of tubular fluid. After completion of late proximal SNGFR collections, collections were also obtained from the early proximal tubule of the same nephron in order to compare values, also under pressure control. The micropuncturist had no foreknowledge of the experimental group (control, ischemic or probucol-treated ischemic) at the time of micropuncture. Tubular pressure measurements were also obtained in 40 randomly chosen tubules in four quadrants of the kidney surface. At least two clearance periods for total GFR were obtained, and blood samples taken for determination of creatinine and probucol levels. After measurements were completed, the kidney was removed and bisected longitudinally. One half was snap-frozen in liquid nitrogen for determination of kidney tissue myeloperoxidase. The other half was fixed in Bouin's, embedded in paraffin, sectioned and stained with periodic acid Schiff (PAS) for histologic study. The histologic sections were examined without knowledge of the treatment given the rat. The percentage and distribution of tubular necrosis present in the sagittal section of the kidneys was assessed by the study of 25-30 non-overlapping adjacent $10 \times$ fields in circumferential bands at the superficial and cortical-medullary levels of the kidney section.

Additional rats ( $n=7$ per group) from ischemic and probucol treated-ischemic groups were evaluated by micropuncture beginning 2 $\mathrm{h}$ after the release of renal artery clamp, using identical experimental techniques in order to assess SNGFR and absolute proximal tubular reabsorption at this earlier period after the ischemic insult.

\section{$\left[{ }^{14} \mathrm{C}\right]$ Inulin microinjection studies}

Early proximal tubular segments were microinjected with nanoliter volumes of $\left[{ }^{14} \mathrm{C}\right]$ inulin to determine the transepithelial leak of inulin in probucol treated control and ischemic animals, and untreated ischemic animals. Isotonic $\mathrm{NaCl}-\mathrm{NaHCO} 3$ was added to $\left[{ }^{14} \mathrm{C}\right]$ inulin for a concentration of $200 \mathrm{cpm} / \mathrm{nl}$, and this fluid stained lightly with FD\&C green. Samples of this fluid were made fresh daily. 5-6-nl vol of this fluid were obtained with a self-filling quartz pipette and placed under oil in the concavity of a microscope slide. These aqueous droplets were then aspirated under direct vision into $7-10-\mu \mathrm{m}$ tip pipettes filled with mineral oil and tipped with a small quantity of oil to prevent evaporation. Alternate samples were delivered into liquid scintillation count- ing vials and utilized as $100 \%$ reference standards for total counts per minute of isotope contained within droplets. Alternate samples were microinjected into proximal tubular segments under concurrent pressure control. The aqueous sample was injected slowly into the tubule with care to prevent injection of oil that might obstruct tubules. Urine samples were collected from the left ureter for two to three consecutive 5 -min periods until radioactive counts in the urine had returned to background levels. Ringer's solution was infused systemically at 1.5 $\mathrm{ml} / \mathrm{h}$ in order to obtain adequate urine flow. $\left[{ }^{3} \mathrm{H}\right]$ Inulin was infused systemically at low concentrations for measurement of total kidney GFR. Urine and plasma samples were submitted to double isotope counting with the appropriate ${ }^{3} \mathrm{H}$ and ${ }^{14} \mathrm{C}$ reference standards and ${ }^{3} \mathrm{H}$ and ${ }^{14} \mathrm{C}$ counts per minute determined in urine samples.

Since $\sim 1,000 \mathrm{cpm}$ of $\left[{ }^{14} \mathrm{C}\right]$ inulin was microinjected into proximal tubules, it was necessary define the effective lower limits of radioactive counting accuracy. Because two to three urine collection periods were assessed and three times background counts per minute are necessary per sample for confidence in a quantitative determination, < $15 \%$ recovery of total $\left[{ }^{14} \mathrm{C}\right]$ inulin injected was defined as the lower limit of quantitative accuracy, based upon the background characteristics of the scintillation counter, the number of collections periods, and the total quantity of radioactive material injected. This finding $(<15 \%$ recovery) could result from $(a)$ tubular obstruction, $(b)$ extremely low tubular flow rates and SNGFR and $(c)$ massive transepithelial backleak of inulin. We cannot discriminate between these various pathophysiologic alternatives.

As tubular obstruction is a contributing factor in IRF, we examined the possibility that osmotic diuresis after ischemia could improve glomerular filtration rate. The effects of mannitol diuresis were evaluated in a separate group of untreated and probucol treated ischemic animals. Urine flow was elevated by the infusion of $10 \%$ mannitol in $0.45 \% \mathrm{NaCl}-\mathrm{NaHCO} 3$ (i.v.) at a rate of $30 \mu \mathrm{l} / \mathrm{min}$. Tubular microinjection studies were conducted as described above, and total kidney GFR was measured.

Myeloperoxidase (MPO) assay. Neutrophils are a potential source of oxygen free radicals $(7,8)$. We examined the levels of MPO, a marker for neutrophils (9) in kidneys from C, I, and IP rats. Kidney tissue was separated into cortex and medulla. Tissue was homogenized in potassium phosphate buffer $(0.01 \mathrm{M})$, centrifuged at $16,000 \mathrm{rpm}$, and rehomogenized. Tubes were freeze-thawed twice and sonicated for $1 \mathrm{~min}$. Samples were mixed with $80 \mathrm{mM}$ potassium phosphate buffer and $16 \mathrm{mM}$ tetramethylbenzidine, and $300 \mathrm{mM} \mathrm{H}_{2} \mathrm{O}_{2}$ was used to initiate the reaction. After a $3-\mathrm{min}$ incubation at $37^{\circ} \mathrm{C}$, the reaction was stopped with the addition of catalase, samples were centrifuged and each supernatant read at $655 \mathrm{~nm}$ (10).

Malondialdehyde (MDA) analysis. To evaluate the extent of lipid peroxidation after $1 \mathrm{~h}$ of renal ischemia and either 15 or $60 \mathrm{~min}$ of reflow, MDA levels were determined in whole kidney homogenates from ischemic rats and control rats. MDA was analyzed by the method of Ohkawa (11).

\section{Oxidizing stimulus experiments}

Preparation of tubular suspension. Male Munich-Wistar rats (150-280 g) were fed for $2 \mathrm{wk}$ with a normal control or a $1 \%$ probucol diet. Animals were anesthetized with Inactin $(100 \mathrm{mg} / \mathrm{kg}$ i.p.). The kidneys were removed, decapsulated, and the cortex dissected free. Cortical tubules were separated from glomeruli and interstitial cells by differential sieving. The tubular suspension was centrifuged at $80 \mathrm{~g}$ for 2 min. The pellet was resuspended in $10 \times$ vol in Krebs-Henseleit buffer (KHS) pH 7.4 with $1 \mathrm{mM}$ alanine and $10 \mathrm{mM}$ butyrate, and centrifuged at $20 \mathrm{~g}$ for $2 \mathrm{~min}$. The pellet was resuspended in $10 \times \mathrm{vol} \mathrm{KHS}$, and again centrifuged at $20 \mathrm{~g}$ for $2 \mathrm{~min}$. The pellet was carefully removed without red blood cell contamination. This procedure yielded a suspension concentrated in renal tubule segments.

Measurement of $M D A$. The protein concentration of the tubule suspension was determined by the Lowry method (12), and adjusted to a concentration of $6 \mathrm{mg}$ protein $/ \mathrm{ml}$. A kinetic study was performed on a concentration series (0-2.4 mM) of tert-butyl hydroperoxide (TBH), 
an oxidizing stimulus (13) resistant to catalase. Proximal tubules are rich in catalase, so it was necessary to use a peroxide that would not be readily degraded. MDA levels in tissues were determined at $0,1,5,10$, 30 , and $60 \mathrm{~min}$ after addition of TBH by a colorimetric assay (11).

Calculations. GFR was determined as previously described (6). The SNGFR was determined by the following equation: $\mathrm{SNGFR}=$ total $\mathrm{cpm}$ of $\left[{ }^{14} \mathrm{C}\right]$ inulin/plasma $\mathrm{cpm} / \mathrm{nl} \cdot$ time of collection, where the plasma counts per minute per nanoliter were corrected for plasma water. The volume of the collected sample was transferred from oiltipped pipettes to a previously calibrated, constant-bore, Pyrex pipette of $\sim 60 \mu \mathrm{m}$ ID. The volume of tubular fluid was determined from length measurements in this pipette. From the total radioactivity count rate and the known volume, the tubular fluid inulin concentration $(T F)$ can be determined, and from the plasma inulin radioactivity $(P)$ (corrected for plasma water), the $T F / P$ inulin ratio. Proximal tubular fractional reabsorption $(F R)$ at the late proximal tubule site can be determined from the $T F / P$ ratio from the following relationship: $F R$ $=(1-P / T F) \times 100 \%$. Absolute proximal reabsorption can be determined from APR $=$ SNGFR $(F R)$. For microinjection studies, total ${ }^{14} \mathrm{C}$ and ${ }^{3} \mathrm{H}$ counts were separated based upon the relative count rates of ${ }^{14} \mathrm{C}$ and ${ }^{3} \mathrm{H}$ standards in ${ }^{14} \mathrm{C}$ and ${ }^{3} \mathrm{H}$ channels. Radioactivity from the left ureter was expressed as percentage of the mean of all $100 \%$ reference standards, which alternated with injected samples.

\section{Statistical methods}

Plasma creatinine values before and $24 \mathrm{~h}$ after renal artery clamping, early and late proximal SNGFR values, and the change in $P_{\mathrm{T}}$ during collection of tubular fluid for SNGFR measurements were analyzed using a paired $t$ test. SNGFR comparison among groups was evaluated by analysis of variance. MDA levels after TBH incubation were analyzed using multiple factor covariance analysis. Other variables were compared using an unpaired $t$ test. As an index of heterogeneity of $P_{\mathrm{T}}$, the SEM for $P_{\mathrm{T}}$ values was divided by the mean $P_{\mathrm{T}}$ in each treatment group.

\section{Results}

\section{Whole kidney function}

There was no difference in growth rate of rats in the three experimental groups, during the 2-wk treatment period before these studies were performed. Clamping of the renal artery for $60 \mathrm{~min}$ followed by $24 \mathrm{~h}$ of reflow caused a significant decrease $(P<0.0001)$ in the GFR of the $I(0.17 \pm 0.04 \mathrm{ml} / \mathrm{min})$ and IP groups $(0.11 \pm 0.06 \mathrm{ml} / \mathrm{min})$ when compared to the shamoperated $\mathrm{C}$ group $(1.39 \pm 0.06 \mathrm{ml} / \mathrm{min})$ as shown in Table I. Urine flow was not significantly different in either the I or IP groups when compared to control rats (Table I). Plasma creatinine levels increased significantly $(P<0.05)$ over $24 \mathrm{~h}$ from $0.5 \pm 0.1$ to $2.5 \pm 0.7 \mathrm{mg} / \mathrm{dl}$ in the ischemic group, and from $0.5 \pm 0.1$ to $3.3 \pm 0.8 \mathrm{mg} / \mathrm{dl}$ in the probucol ischemic group. Creatinine levels did not change significantly in control animals submitted to sham renal artery clamping (Fig. 2). When plasma creatinine levels were measured on day 0 through 7 after ischemia, there was no significant difference between I

Table I. The Whole Kidney GFR Measured by Clearance of $\left[{ }^{3} \mathrm{H}\right]$ Inulin, the Urine Flow Rate (i), the Late Proximal SNGFR, and Absolute Collection of Proximal Reabsorption (APR) Are Presented for the Three Treatment Groups, with the Tubular Pressure P before and during Tubular Fluid Collection at $24 \mathrm{H}$ after $1 \mathrm{H}$ of Renal Ischemia or Sham Ischemia

\begin{tabular}{|c|c|c|c|c|c|c|c|}
\hline Treatment & Rat & GFR & $\dot{\mathbf{v}}$ & Late proximal SNGFR & APR & $P_{\mathrm{T}}$ (before) & $P_{\mathrm{T}}$ (during collection) \\
\hline & & $\mathrm{ml} / \mathrm{min}$ & $\mu l / \min$ & $\mathrm{nl} / \mathrm{min}$ & $\mathrm{nl} / \mathrm{min}$ & $m m H g$ & $\mathrm{mmHg}$ \\
\hline \multirow[t]{8}{*}{ Control } & 1 & $1.61 \pm 0.07$ & $5.2 \pm 0.5$ & $61.8 \pm 5.4$ & $17.6 \pm 3.2$ & $19.3 \pm 1.0$ & $14.5 \pm 1.8$ \\
\hline & 2 & $1.14 \pm 0.02$ & $4.7 \pm 0.1$ & $28.7 \pm 4.4$ & $9.6 \pm 3.3$ & $14.3 \pm 1.3$ & $13.3 \pm 1.0$ \\
\hline & 3 & $1.41 \pm 0.01$ & $3.6 \pm 0.1$ & $48.9 \pm 4.7$ & $17.8 \pm 1.0$ & $17.6 \pm 1.5$ & $14.6 \pm 2.2$ \\
\hline & 4 & $1.40 \pm 0.22$ & $6.0 \pm 0.1$ & $36.2 \pm 0.4$ & $7.9 \pm 1.4$ & $16.3 \pm 1.3$ & $15.3 \pm 1.7$ \\
\hline & 5 & $1.25 \pm 0.12$ & $4.6 \pm 0.4$ & $41.8 \pm 3.5$ & $13.0 \pm 1.2$ & $12.3 \pm 1.0$ & $10.5 \pm 1.0$ \\
\hline & 6 & $1.52 \pm 0.02$ & $6.0 \pm 0.2$ & $52.9 \pm 2.3$ & $20.6 \pm 1.1$ & $16.8 \pm 1.3$ & $15.3 \pm 1.1$ \\
\hline & 7 & $1.43 \pm 0.50$ & $6.0 \pm 0.2$ & $46.4 \pm 0.8$ & $16.9 \pm 1.5$ & $13.8 \pm 0.6$ & $13.8 \pm 0.6$ \\
\hline & $\overline{\mathbf{x}} \pm \mathrm{SE}$ & $1.39 \pm 0.06$ & $5.2 \pm 0.1$ & $45.2 \pm 4.1$ & $14.8 \pm 1.8$ & $15.9 \pm 0.9$ & $13.9 \pm 0.6^{*}$ \\
\hline \multirow[t]{8}{*}{ Ischemic } & 1 & $0.33 \pm 0.02$ & $3.6 \pm 0.3$ & $10.3 \pm 5.9$ & $3.5 \pm 3.0$ & $15.5 \pm 1.0$ & $10.5 \pm 1.3$ \\
\hline & 2 & $0.17 \pm 0.05$ & $10.0 \pm 2.8$ & $26.1 \pm 6.4$ & $9.5 \pm 1.4$ & $18.3 \pm 4.7$ & $17.3 \pm 3.3$ \\
\hline & 3 & $0.24 \pm 0.03$ & $7.4 \pm 1.7$ & $16.0 \pm 11.3$ & $8.4 \pm 3.5$ & $18.7 \pm 3.8$ & $17.7 \pm 3.8$ \\
\hline & 4 & $0.16 \pm 0.01$ & $8.1 \pm 0.2$ & $23.7 \pm 3.9$ & $7.7 \pm 1.7$ & $26.3 \pm 2.2$ & $23.5 \pm 2.8$ \\
\hline & 5 & $0.08 \pm 0.01$ & $5.7 \pm 0.4$ & $13.8 \pm 2.6$ & $6.2 \pm 1.1$ & $17.6 \pm 1.3$ & $18.6 \pm 1.5$ \\
\hline & 6 & $0.19 \pm 0.01$ & $9.5 \pm 0.4$ & $14.2 \pm 6.1$ & $5.4 \pm 2.2$ & $25.3 \pm 2.0$ & $20.8 \pm 2.1$ \\
\hline & 7 & $0.03 \pm 0.01$ & $3.2 \pm 0.1$ & $2.5 \pm 1.4$ & $2.2 \pm 1.1$ & $22.0 \pm 2.8$ & $23.3 \pm 3.2$ \\
\hline & $\overline{\mathrm{x}} \pm \mathrm{SE}$ & $0.17 \pm 0.04^{*}$ & $6.8 \pm 1.0$ & $15.2 \pm 3.0^{* *} \downarrow$ & $6.1 \pm 1.0^{*}$ & $20.5 \pm 1.6$ & $18.8 \pm 1.7$ \\
\hline \multirow{8}{*}{$\begin{array}{l}\text { Probucol } \\
\text { ischemic }\end{array}$} & 1 & $0.35 \pm 0.02$ & $2.9 \pm 0.8$ & $26.6 \pm 5.0$ & $8.3 \pm 2.0$ & $21.0 \pm 2.9$ & $21.0 \pm 4.7$ \\
\hline & 2 & $0.03 \pm 0.01$ & $4.3 \pm 0.2$ & $37.0 \pm 8.4$ & $5.0 \pm 1.3$ & $11.6 \pm 2.9$ & $14.1 \pm 0.4$ \\
\hline & 3 & $0.06 \pm 0.01$ & $13.5 \pm 1.8$ & $24.3 \pm 14.9$ & $5.6 \pm 3.7$ & $19.0 \pm 1.0$ & $15.8 \pm 0.2$ \\
\hline & 4 & $0.03 \pm 0.01$ & $5.7 \pm 0.3$ & $29.1 \pm 4.1$ & $5.2 \pm 2.1$ & $21.1 \pm 5.7$ & $17.8 \pm 2.9$ \\
\hline & 5 & $0.19 \pm 0.01$ & $17.1 \pm 0.8$ & $21.9 \pm 5.7$ & $3.8 \pm 1.0$ & $19.4 \pm 1.5$ & $20.4 \pm 0.9$ \\
\hline & 6 & $0.01 \pm 0.01$ & $0.1 \pm 0.1$ & $41.6 \pm 2.0$ & $10.4 \pm 2.1$ & $21.5 \pm 1.5$ & $18.0 \pm 1.4$ \\
\hline & 7 & $-\quad-$ & -- & $16.0 \pm 8.4$ & $4.7 \pm 2.8$ & $18.8 \pm 0.5$ & $19.3 \pm 1.8$ \\
\hline & $\overline{\mathbf{x}} \pm \mathrm{SE}$ & $0.11 \pm 0.06^{*}$ & $7.3 \pm 2.7$ & $28.1 \pm 3.3^{*}$ & $5.9 \pm 0.8^{*}$ & $18.9 \pm 1.3$ & $18.1 \pm 0.9$ \\
\hline
\end{tabular}

Mean \pm SE are given. ${ }^{*}=P<0.5,{ }^{* *}=P<.001$ vs. control, $\downarrow=P<.05$ vs probucol ischemic. 


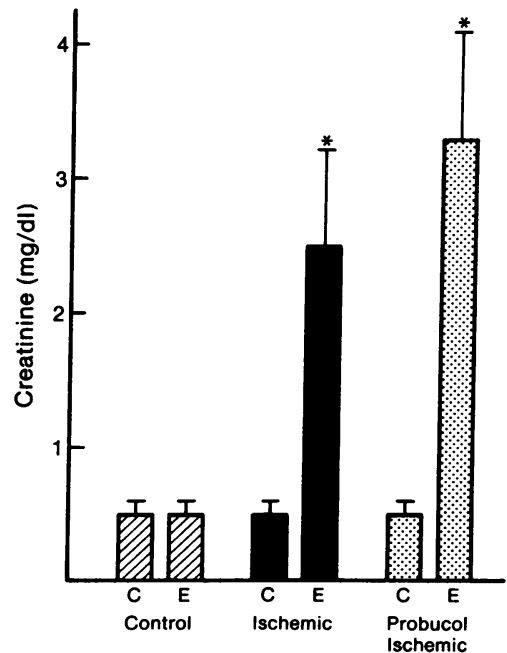

Figure 2. The effect of sham surgery or ischemia on serum creatinine. Serum creatinine levels were measured before (C) and $24 \mathrm{~h}$ after (E) sham surgery in control animals, or 1 h of renal artery occlusion in ischemic animals or probucoltreated ischemic animals. Means $\pm S E$ are given. ${ }^{*} P<0.05$ vs. $C$.

and IP groups. Protective effects of the antioxidant probucol were not apparent from examining the whole kidney function data.

\section{Micropuncture evaluation}

In addition to examining whole kidney function after ischemia and reflow, we evaluated function at the single nephron level. The group of rats that underwent $60 \mathrm{~min}$ of renal ischemia and $24 \mathrm{~h}$ of reflow without pretreatment with probucol, exhibited a significantly lower SNGFR $(15.2 \pm 3.0 \mathrm{nl} / \mathrm{min})$ collected from the late proximal tubule than either the control $(45.2 \pm 4.1)$ group or the probucol-treated group $(28.1 \pm 3.3)$. These results are summarized in Table I. Collections of tubular fluid for SNGFR measurements were carefully pressure controlled. Values for $P_{\mathrm{T}}$ before and during tubular fluid collections are presented in Table I. There was no significant change in tubular pressure during the collection of tubular fluid when compared to the tubular pressure before the collection in the $I$ and IP groups. Differences in SNGFR among the different treatment groups were not artifactually induced by changes in $P_{\mathrm{T}}$ during collections. Pretreatment with probucol did not totally prevent a reduction in SNGFR when compared to the control group, but did significantly ameliorate the change induced 24 $h$ after ischemia. The value for SNGFR for the probucoltreated animals was $\sim 100 \%$ greater than the ischemic animals. This suggests that probucol exerted a beneficial effect on SNGFR that was not attributable to an artifactual reduction in $P_{\mathrm{T}}$ by the tubular fluid collection technique. The distribution of SNGFR within each group is depicted graphically in Fig. 3.

\begin{tabular}{|c|c|c|c|c|}
\hline & & Control & $\begin{array}{l}\text { Untreated } \\
\text { Ischemic }\end{array}$ & $\begin{array}{l}\text { Probucol } \\
\text { Ischemic }\end{array}$ \\
\hline $\begin{array}{c}\text { Single } \\
\text { Nephron } \\
\text { GFR }\end{array}$ & $\begin{array}{r}0-10 \\
11-20 \\
21-30 \\
31-40 \\
41-50 \\
51-60 \\
61-70\end{array}$ & $\begin{array}{l}\cdots \\
\cdots \cdots \cdots \\
\cdots \cdots \\
\cdots\end{array}$ & $\begin{array}{l}\bullet \cdots \cdots \cdots \cdots \cdots \\
\cdots \cdots \cdots \\
\cdots \cdots \cdots \\
\bullet \cdots\end{array}$ & $\begin{array}{l}\cdots \\
\cdots \cdots \\
\cdots \cdots \\
\cdots \cdots \\
\bullet \cdots \\
\bullet\end{array}$ \\
\hline
\end{tabular}

Figure 3. The distribution of SNGFR values in control, untreated ischemic, and probucol-treated ischemic groups is depicted individually.
These data represent a totally random selection of surface tubules for assessment of SNGFR and absolute proximal reabsorption.

$P_{\mathrm{T}}$ changed significantly during early proximal tubular fluid collection in each treatment group. It was impossible to maintain a constant $P_{\mathrm{T}}$ during early proximal tubular collections, in all likelihood due to the proximity to Bowman's space and the absence of a Starling resistor effect provided by a significant length of proximal tubule. Early proximal SNGFR values obtained in the same nephrons were significantly lower $(P<0.01)$ in I $(23.1 \pm 4.6)$ and IP $(28.5 \pm 2.6)$ animals than in C $(48.7 \pm 4.7)$ animals. Early and late proximal collections produced nearly identical values for SNGFR in the IP group.

Because tubular obstruction is a significant contributing factor to IRF, we examined the possibility that $\boldsymbol{P}_{\mathrm{T}}$ might be altered after ischemia and reflow. Mean tubular pressures were not significantly higher in the ischemic or probucol-treated ischemic group than in control animals (Fig. 4). When the SEM for $P_{\mathrm{T}}$ values was divided by the mean $P_{\mathrm{T}}$ in each group as an index of heterogeneity, the value for control was $7.7 \%$, for ischemics was $10.1 \%$, and for probucol ischemics was $11.8 \%$. Heterogeneity of $P_{\mathrm{T}}$ increased slightly after ischemia.

Ischemia and reperfusion was associated with a significant decrease in APR of $\mathrm{NaCl}$ and water in both the probucol treated and untreated ischemic groups. The mean value for APR in control animals was $14.8 \pm 1.8 \mathrm{nl} / \mathrm{min}$. In the ischemic group (6.1 \pm 1.0$)$ as well as the probucol-treated ischemic group $(5.9 \pm 0.8)$, the mean value for APR was decreased (Table I). The $T F / P$ for inulin was somewhat increased in the ischemic group $(1.88 \pm 0.13)$ when compared with controls $(1.49 \pm 0.05)$, but was decreased in the probucol group (1.29 \pm 0.04$)$. In the probucol group, changes in SNGFR and APR were dissociated; in spite of the increased SNGFR, there was no improvement in APR. The APR fell in proportion to SNGFR in the untreated ischemic group, but when SNGFR was increased in probucol-treated ischemic rats, APR remained quite low and did not rise with SNGFR. Therefore, APR in the I group may have represented a minimum estimate of true proximal tubular reabsorptive capacity, limited by the very low values for SNGFR in this group. After $2 \mathrm{~h}$ of reflow, SNGFR was significantly higher in IP $(36.0 \pm 3.1)$ than in I $(20.8 \pm 2.7)$ rats (Fig. 5). These results are similar to the SNGFR values recorded after $24 \mathrm{~h}$ reflow. When APR was measured after $2 \mathrm{~h}$ reperfusion, values in IP rats $(11.6 \pm 1.3)$ were significantly higher than in I rats (7.4 \pm 1.1 ). APR values were normal in IP rats at $2 \mathrm{~h}$ reflow but declined significantly over $24 \mathrm{~h}$. These results suggest an early protective effect of probucol on the function of the proximal tubules.

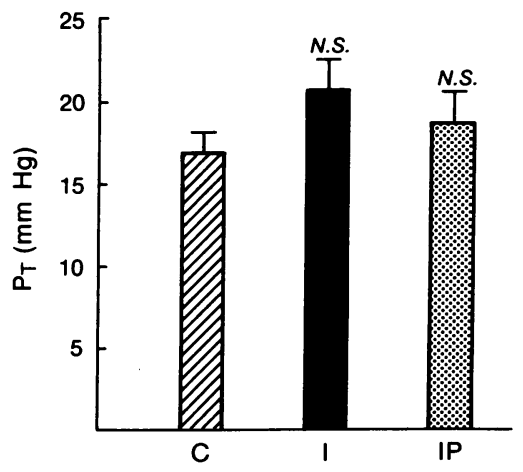

Figure 4. Tubular $\left(P_{\mathrm{T}}\right)$ pressures $(\overline{\mathrm{x}} \pm \mathrm{SE})$ from 40 randomly chosen tubules from each rat are shown for control (C), untreated ischemic (I), probucol-treated ischemic (IP) animals. N.S., not significantly different from control. 


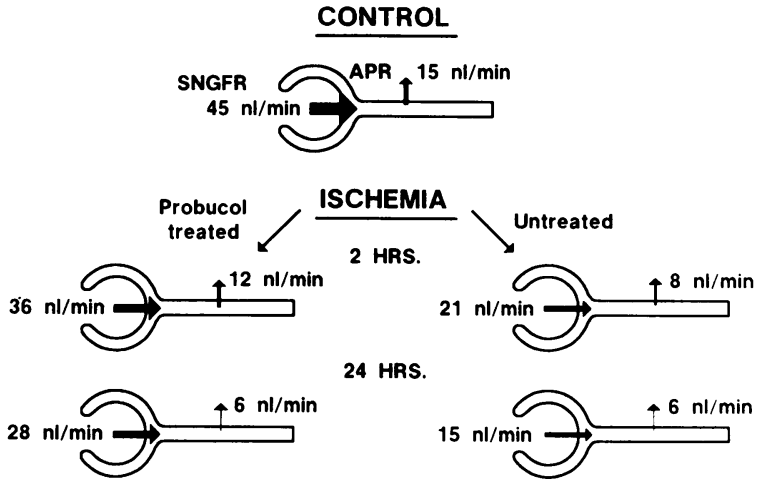

Figure 5. The progression of SNGFR and APR changes at 2 and 24 $\mathrm{h}$ after $1 \mathrm{~h}$ of ischemia are shown for untreated and probucol-treated rats.

\section{Tubular injection of $\left[{ }^{14} \mathrm{C}\right]$ inulin}

The kidney GFR was reduced to a greater extent than the mean SNGFR in both I and IP groups. A logical reason for this finding was major backleak of filtered inulin across damaged epithelia. The measurement of recovery of microinjected $\left[{ }^{14} \mathrm{C}\right]$ inulin permitted the evaluation of tubular backleak and an assessment of the degree of tubular obstruction and/or extremely low tubular flow rate. Urine was collected during two to three consecutive 5-min periods and the vast majority of counts were recovered during the first collection period for all treatment groups, suggesting that recirculation of $\left[{ }^{14} \mathrm{C}\right]$ inulin was a small component of the total inulin excreted. All microinjections were rigidly pressure controlled. In control animals, the mean recovery was $98.9 \pm 2.7 \%$ (Fig. 6). In probucoltreated animals that had undergone renal ischemia, the mean recovery was $33.1 \pm 6.9 \%$. Inulin recoveries of $15-75 \%$ were present in $36 \%$ of tubules in IP animals. These results are

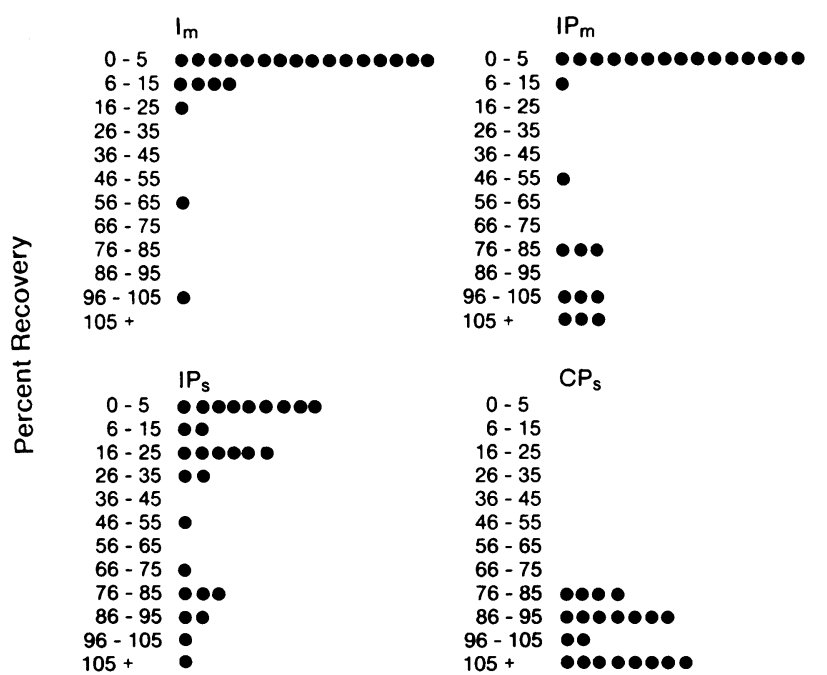

Figure 6. Percent recovery of $\left[{ }^{14} \mathrm{C}\right]$ inulin microinjected into proximal tubules under pressure controlled conditions is presented for untreated ischemic (Im) and probucol-treated ischemic (IPm) animals that underwent mannitol diuresis during the recovery studies, and probucol-treated control (CPs) and ischemic (IPs) animals that underwent mild saline diuresis during the recovery studies, $24 \mathrm{~h}$ after ischemia. compatible with backleak of inulin across the tubular epithelium. There was essentially no recovery of inulin $(<15 \%)$ in $39 \%$ of tubules. The value of $15 \%$ was determined by the lowest limits of radioactive counting accuracy. This evaluation was a quantitative experimental judgment rather than an anatomical/physiologic evaluation. Those tubules that exhibited $<15 \%$ inulin excretion may by clearance evaluation have contributed significantly to the GFR since inulin is infused continuously in those studies.

Since tubular obstruction is an important factor in ischemic renal failure, we examined the possibility that diuresis with mannitol might improve glomerular filtration by relieving tubular obstruction. $P_{\mathrm{T}}$ was nearly identical in mannitol and isotonic $\mathrm{NaCl} / \mathrm{HCO}_{3}$ infused rats. We measured recovery of $\left[{ }^{14} \mathrm{C}\right]$ inulin injected into tubules of probucol-treated ischemic animals and untreated ischemic animals. The mean recovery of inulin from untreated ischemic (Im) animals was $10.0 \pm 5.0 \%$, and from treated ischemic (IPm) animals was $35.7 \pm 9.2 \%$. Very low recoveries $(<15 \%)$ were observed in $87 \%$ of tubules in untreated ischemic animals. This frequency of poor inulin recovery correlates with the low SNGFR (15.2 \pm 3.0 $\mathrm{nl} / \mathrm{min}$ ) observed in this group. Mannitol diuresis did not improve the whole kidney GFR or the recovery of $\left[{ }^{14} \mathrm{C}\right]$ inulin in either the untreated or probucol treated ischemic groups. The results from these microinjection studies suggest that both tubular backleak and tubular obstruction are important contributing factors in ischemic renal failure. Probucol treatment appears to decrease the number of nephrons exhibiting low SNGFR, but significant tubular backleak of inulin persisted.

\section{Structural evaluation of kidneys}

Gross appearance. Control kidneys were firm and reddish brown in color when viewed under the microscope at the time of micropuncture. Tubular flow was brisk and no cellular debris was noted in the tubules. Untreated ischemic kidneys were mottled in appearance and had a soft spongy consistency. Many tubules exhibited very slow flow when dye was injected into proximal tubules, and debris was visible in some tubules. Areas were present in which all the tubules were very pale and many tubules were thin walled in appearance, suggesting tubular dilatation and obstruction. When kidneys were bisected, the entire medulla appeared congested. The micropuncture surface of probucol treated ischemic kidneys was similar in appearance to untreated ischemic kidneys, except that the pale zones containing thin walled tubules with low flow were not evident. In some tubules, slow tubular flow was present and debris was also noted in some tubules. The appearance of the bisected probucol kidney surface was intermediate between the control and I groups. The "red zone" was present in probucol rats but the outer medulla was less congested than in the I group.

\section{Histologic evaluation}

The histologic appearance of the kidneys at $24 \mathrm{~h}$ after ischemia was that of variable and often extensive tubular necrosis, large numbers of intraluminal casts and cellular debris, and congestion of peritubular capillaries. The extent and severity of the lesions varied somewhat from one region of the section to another, with casts and tubular debris involving more than $90 \%$ of tubules in some areas (Fig. $7 A$ ). In ischemic kidneys with massive necrosis, pigments from lysed red blood cells in peritubular capillaries was present and prominent PAS posi- 

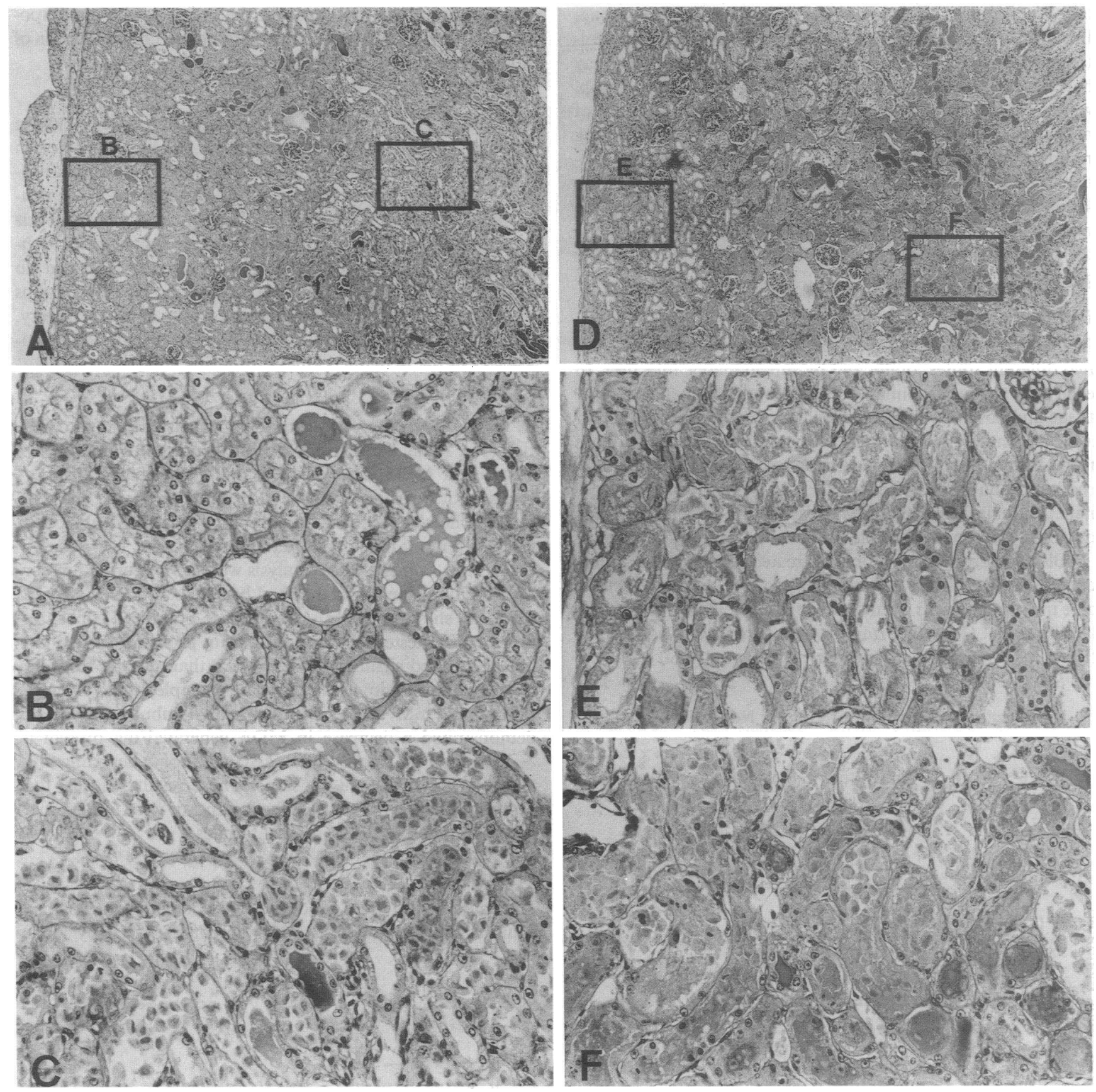

Figure 7. The histologic changes found in untreated ischemic $(A-C)$ and probucol-treated ischemic rats $(D-F)$ are shown. A much greater degree of necrosis of renal tubular cells (loss of nuclear detail and detachment from the tubular basement membrane) was noted in superficial cortex $(E)$ in the probucol-treated ischemic group than in the

tive droplets were found in the cytoplasm of tubules at the papillary tip (Fig. 7 B). The glomeruli appeared to be generally well preserved, but sometimes gave the impression of being less distended than usual, producing angulation in the glomerular capillary walls (Fig. $7 \mathrm{C}$ ). The glomerular cellularity was similar to nonischemic controls. In kidneys from IP rats with areas of massive tubular necrosis, vacuoles filled with PAS positive material could be found in glomerular cells (Fig. $7 \mathrm{D}$ ). A small increase in polymorphonuclear leukocytes was found in glomeruli from IP rats, however, it was unusual to find more

untreated ischemic group $(B)$. The degree of necrotic changes and compaction of necrotic cells within the tubular lumens also appeared somewhat greater in the probucol group when the deeper areas of the cortex and juxtamedullary junction were compared ( $C$ and $F$ ). Original magnification $A$ and $D \times 50, \times 312$, PAS stain.

than three of these cells per glomerular cross-section (Fig. $7 E$ ). Control rats of the same age average less than one polymorphonuclear leukocyte per glomerular cross-section. The changes noted among the rats of the two ischemic treatment groups were similar in kind, but different in extent and distribution particularly with regard to tubular necrosis. Considerably more extensive tubular necrosis was evident in the superficial cortex in the probucol-treated group than in the untreated ischemic control group (Fig. 7 F). Somewhat more necrosis of tubules in the deeper layers of the cortical medul- 
lary junction was also present in the probucol-treated group. Intraluminal tubular debris was more obvious in probucoltreated animals in the deeper zones of the kidney. This may have resulted from the higher tubular flow rates in this group, which moved cellular debris into more distal segments of nephrons. These changes were semiquantitated by comparing multiple adjacent $10 \times$ fields in the superficial cortex and cortical medullary junction bands of the sagittal sections of the kidneys. The results are presented as a histogram showing the number of $10 \times$ fields with increasing percentages of necrotic tubules for the probucol-treated and untreated ischemic groups (Fig. 8).

\section{Probucol assay}

In the experiments described, probucol was administered by two different routes; orally and by subcutaneous injection. In each case, we found similar levels of probucol $(\mathrm{P})$ in the kidney tissue and observed similar beneficial effects on renal function after ischemia. We measured probucol levels in the kidneys from animals in all three treatment groups. The animals receiving probucol exhibited $6.7 \pm 0.5 \mu \mathrm{g} \mathrm{P} / \mathrm{ml}$ kidney homogenate. Animals from the untreated ischemic and control groups demonstrated no measurable probucol levels in their kidneys.

Blood analysis from probucol-treated rats revealed probucol present in plasma $(0.95 \mu \mathrm{g} \mathrm{P} / \mathrm{mg}$ protein) with none detectable in red blood cells. Renal tubule probucol levels were similar to plasma (1.11 $\mu \mathrm{g} \mathrm{P} / \mathrm{mg}$ protein). Probucol was concentrated in renal proximal tubular membranes $(2.60 \mu \mathrm{g} P / \mathrm{mg}$

\section{Histological Assessment of Tubular Necrosis}

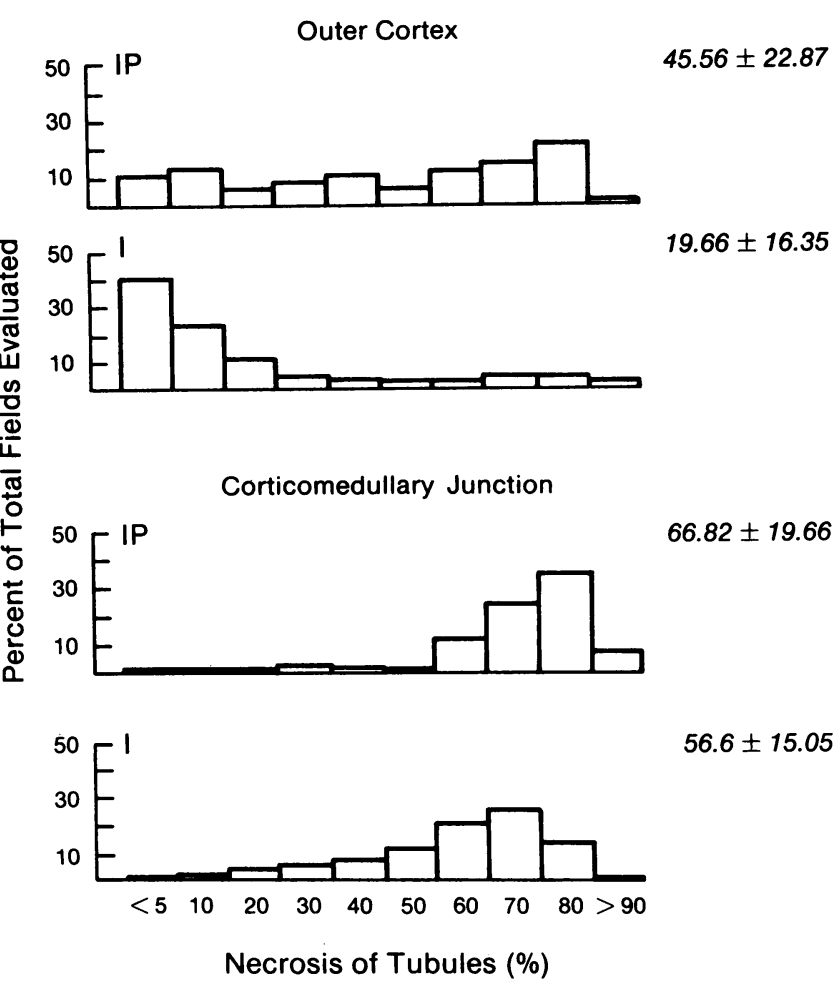

Figure 8. Histological assessment of tubular necrosis. Adjacent $10 \times$ fields in the outer cortex and corticomedullary junction were evaluated for extent of tubular necrosis for probucol-treated ischemic (IP) and untreated ischemic (I) animals. The mean \pm SD percent necrosis for all fields evaluated is shown on the right side of the graph. protein), while no probucol was detectable in the renal lipoprotein fraction, suggesting that there was no contamination of homogenates by plasma lipoproteins.

\section{MPO assay}

Polymorphonuclear leukocytes are a known source of oxygen free radicals, and neutrophil depletion has been reported to ameliorate phorbol myristate acetate-induced glomerular injury in experimental animals (14). MPO is present in the neutrophil in exceptionally high concentrations (8). MPO levels were measured in cortex and outer medulla from control, ischemic, and probucol-treated ischemic animals. We found no significant difference in MPO activity among the three groups either 2 or $24 \mathrm{~h}$ after renal artery clamping or sham surgery. There was no evidence of increased polymorphonuclear leukocytes in the ischemic kidneys from this analysis.

\section{MDA analysis}

In vivo studies. MDA is one of the products of lipid peroxidation (15). Renal tissue from ischemic and control animals was assayed for MDA. There was no significant change in MDA levels in kidneys harvested after $1 \mathrm{~h}$ of reflow $(54.3 \pm 10.8 \mathrm{~nm} / \mathrm{g}$ tissue) following ischemia, when compared to control kidneys $(36.7 \pm 4.5 \mathrm{~nm} / \mathrm{g}$ tissue), or after $15 \mathrm{~min}$ reflow.

In vitro studies. MDA analysis was also performed in cortical tubule suspensions that had been exposed to an in vitro oxidizing stimulus, TBH. At each time point and concentration of TBH tested, renal tubules from probucol-treated animals generated significantly less MDA than tubules from untreated control animals (Fig. 9). For example, at a concentration of $1.2 \mathrm{mM} \mathrm{TBH}$, probucol-treated tubule suspensions demonstrated a decrease in MDA generated $/ \mathrm{mg}$ protein of $\sim 50 \%$ at all time points after $\mathrm{TBH}$ addition when compared with control tubule suspensions. These results suggest that probucol exerts a protective effect against in vitro lipid peroxidation initiated by TBH in renal tubules.

\section{Discussion}

The acute renal failure produced by ischemia and reflow is a clinical and experimental syndrome characterized by major

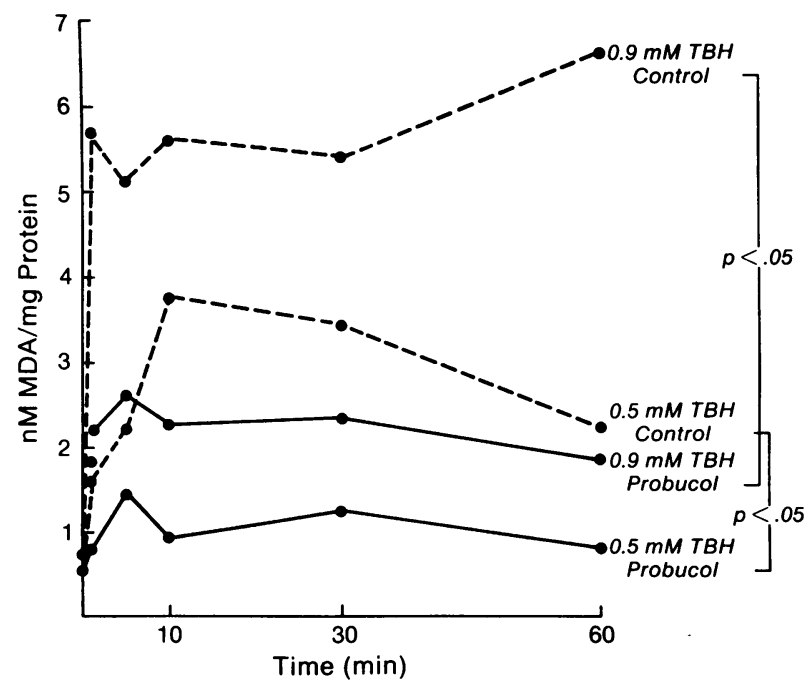

Figure 9. MDA generation by renal tubules. The effect of probucol pretreatment on in vitro generation of MDA, a marker of lipid peroxidation, by renal tubules in response to TBH, an oxidizing stimulus is shown. 
reductions in glomerular filtration rate, extensive tubular damage, tubular cell necrosis, glomerular injury, and signs of tubular obstruction with cell debris (16-19). The complex relationship that exists between tubular damage and the reductions in GFR has not been fully defined. Tubular damage may influence whole kidney GFR by producing tubular obstruction and/or extensive tubular backleak of solutes across the damaged epithelia. However, ischemia and reperfusion may also have more direct or even indirect effects upon glomerular function. Much of this tubular and glomerular dysfunction has been postulated to occur during the reperfusion period following anoxia, and generation of oxygen free radicals has been postulated as one of the factors contributing to this reperfusion injury.

Recent studies utilizing SOD and allopurinol as therapy have suggested an important role for the generation of oxygen free radicals in the pathogenesis of acute renal failure (2). Paller and coauthors demonstrated that administration of a large molecular weight substance such as SOD improved whole kidney GFR in an experimental model of acute renal failure $24 \mathrm{~h}$ after total renal ischemia. This treatment did not restore GFR to normal, implying major persisting renal abnormalities in spite of therapy directed toward the elimination of oxygen free radicals. The goals of the current studies were two in number. First, we reasoned that administration of a nontoxic antioxidant, probucol, which is lipophilic and presumably capable of achieving high concentrations in renal lipid membranes, might provide more complete protection against ischemia and reflow and the consequent generation of reactive oxygen species than some of the other antioxidants. Second, no specific information has been provided as to the mechanism whereby modification or prevention of the effects of oxygen radicals leads to improvement in renal function $24 \mathrm{~h}$ after $1 \mathrm{~h}$ of total ischemia and reflow. Do antioxidants prevent tubular injury or is there some other beneficial influence upon GFR and the factors contributing to kidney GFR that is separate and independent of the degree of tubular injury? The current results suggest that probucol therapy provides an initial beneficial effect on SNGFR and proximal tubular reabsorption within a few hours after ischemia, but at $24 \mathrm{~h}$ after the ischemic insult, nephron filtration rates remain elevated above values in untreated rats, but the absolute proximal reabsorption declines to significantly subnormal values.

The major alteration in renal function that results from probucol pretreatment after the ischemic insult is the major improvement in nephron filtration rate. The assessment of nephron filtration rate in all groups was conducted under carefully pressure controlled conditions, and tubules for assessment were selected in a highly random fashion to reflect accurately the heterogeneity that might exist. Although probucoltreated rats exhibited a nephron filtration rate that was $60-65 \%$ of normal control rats, the GFR in this group remained quite low. The backleak of inulin across damaged tubular epithelia may explain the discrepancy between changes in SNGFR and GFR. In the untreated ischemic group, the changes in SNGFR were more closely correlated with changes in GFR requiring only modest further transepithelial backleak of inulin. Conger has reported SNGFR values closely correlated with predicted values from whole kidney inulin clearances in a norepinephrine induced model of ischemic renal failure (20). However, the discrepancy between changes in SNGFR and GFR in the probucol-treated ischemic group was more difficult to explain. The improvement in SNGFR pro- vided by probucol in ischemic rats was in major part the result of decreasing the percentage of nephrons with poor function, either low SNGFR or $<15 \%$ recovery of microinjected inulin. The latter assessment is qualitatively different in that low inulin recovery could have been the result of either very low SNGFR and tubular flow rates or tubular obstruction. The most logical reason for the disparity between changes in SNGFR and GFR in the probucol group was a greater backleak of inulin from those nephrons with a more reasonable SNGFR.

Microinjection studies provided some insights into the mechanisms of SNGFR and GFR reduction in ischemic and probucol ischemic rats. These data are particularly instructive if the distribution of SNGFR and microinjection recoveries are compared in Figs. 3 and 6. In the I group, mean recovery of acutely microinjected inulin was $\sim 10 \%$ compared with $\sim 36 \%$ in probucol-treated rats. However, this very low value in I rats is due to the fact that $87 \%$ of microinjected tubules fell below the level of detectability of radioactive counting accuracy ( $<15 \%$ injected inulin). When the distribution of SNGFR in Fig. 3 is examined, it is obvious that with steady state inulin infusions, this large number of low flow and/or partially obstructed tubules do exhibit low but significant values for SNGFR ( <15 nl/min) and therefore contribute to the total GFR. The actual degree of inulin backleak in the few nephrons with higher flow rates in the I group cannot be accurately assessed. Since only one-third of the nephrons in the probucol-treated group exhibited very low flow rates and/ or obstruction by microinjection evaluation, the degree of inulin backleak could be more accurately evaluated. The degree of backleak of inulin was significant in most of these higher flow nephrons in probucol treated rats. Although probucol treatment decreased the number of nephrons exhibiting very low SNGFR and tubular flow rates (Figs. 3 and 6), the degree of inulin backleak remained great and possibly greater than in I rats. The more extensive tubular necrosis observed at $24 \mathrm{~h}$ in the probucol treated animals may account in part for this high rate of inulin backleak.

We measured the effects of probucol on MDA generation by proximal tubules exposed to an in vitro oxidizing stimulus, and found a significant protective effect of probucol on MDA production that can be interpreted as a reduction in lipid peroxidation. Probucol is concentrated in renal tubular membranes and these in vitro results suggest that probucol acts as an effective antioxidant in this setting. MDA is a good indicator of the rate of lipid perioxidation in vitro, but because of the transient nature of lipid hydroperoxides and their products, and the excretory pathways of urine and blood it is less dependable in vivo (15). We were unable to detect a significant increase in MDA generation after ischemia and reflow in vivo in untreated rats, which prevented the examination of a probucol effect on MDA production in this model.

The beneficial effects of probucol are presumably related to activity as an antioxidant agent, but could also derive from a decrease in production of oxygen radicals. Neutrophils are a potential source of oxygen free radicals $(7,8)$. Linas et al. have reported that addition of neutrophils to the perfusate accentuate ischemic-reperfusion injury in the isolated perfused kidney (21). Neutrophils may contribute to injury in ischemic renal failure but the data from the above studies suggest that they are not likely as the primary source of oxygen free radicals in renal ischemic injury.

Although administration of the antioxidant probucol did 
not produce complete protection against acute ischemic renal failure after $24 \mathrm{~h}$ of reflow, the aggregate results of this study do provide considerable insights into the pathogenesis of this disorder. Evaluation of nephron filtration rate and absolute proximal reabsorptive rates in probucol treated animals at both 2 and $24 \mathrm{~h}$ of reflow reveals that at both time periods, nephron filtration rate is significantly greater than in untreated ischemic animals. However, only at $2 \mathrm{~h}$ did we observe essentially normal rates of tubular reabsorption within the proximal tubule. At $24 \mathrm{~h}$, absolute proximal reabsorption had decreased to values that were at or below $50 \%$ of normal. This finding coincided with a greater degree of necrosis of tubules, primarily localized to the proximal tubule, compared to untreated ischemic animals. A logical conclusion from these data is that the antioxidant provides protection against the damaging effects of oxygen radicals generated early during the reperfusion process. After this early beneficial effect, the increased filtered load at the glomerulus resulted in an increase in transport dependent oxygen demand without alleviating all of the abnormalities in cellular function during this ensuing 24-h period. Such a conclusion would require that the resulting proximal tubular dysfunction and necrosis was a consequence of the initial insult and the degree of oxygen dependent transport demands placed upon the nephron. This formulation would fit well with the hypothesis of Brezis et al. (22). These investigators have reported that reduction in filtration rate and a decrease in epithelial transport rate in the medullary thick ascending limb of the loop of Henle may ameliorate histologic damage in ischemic models in the isolated perfused kidney. Inhibition of solute transport with furosemide or ouabain can protect the medullary thick ascending limb from necrosis and histologic damage after ischemia.

The results of these studies suggest that $(a)$ early in the reperfusion process following ischemia injury, administration of antioxidants such as probucol significantly improves nephron filtration rate and proximal tubular reabsorption compared with untreated ischemic rats. $(b)$ The results of this study and those of Brezis and co-workers (22) raise the interesting possibility that maneuvers that increase nephron filtration rate without completely alleviating tubular damage may promote further tubular injury and cell necrosis during the ensuing $24 \mathrm{~h}$ by placing increased metabolic demands upon damaged reabsorbing epithelia. (c) These studies reaffirm and extend the previously observations in ischemic renal failure that multiple factors contribute to both glomerular and tubular dysfunction during periods of ischemia and reperfusion. These studies emphasize the intimate interrelation of glomerular and tubular function in the postischemic kidney.

\section{Acknowledgments}

The authors thank Dr. R. P. O'Connor and Dr. H. D. Humes for information on the use of TBH as an oxidative stress.

Portions of this work supported by the Medical Research Service of the Veterans Administration and grants from the National Institutes of Health (DK-28602, DK-33692, PHS-5T32 HL-07261, DKL-20043, and AI-07007). This is publication IMM-4824 from the Department of Immunology, Research Institute of Scripps Clinic.

\section{References}

1. Arendshorst, W. J., W. F. Finn, and C. W. Gottschalk. 1975. Pathogenesis of acute renal failure following renal ischemia in the rat. Circ. Res. 37:558-568.
2. Paller, M. S., J. R. Moidal, and T. F. Ferris. 1984. Oxygen free radicals in ischemic acute renal failure in the rat. J. Clin. Invest. 74:1156-1164.

3. Barnhart, J. W., D. J. Rytter, and J. A. Molello. 1977. An overview of the biochemical pharmacology of probucol. Lipids. 12:29-33.

4. Parthasarathy, S., S. G. Young, J. L. Witztum, R. C. Pittman, and D. Steinberg. 1986. Probucol inhibits oxidative modification of low density lipoprotein. J. Clin. Invest. 77:641-644.

5. Satonin, D. K., and J. E. Coutant. 1986. Comparison of gas chromatography and high-performance liquid chromatography for the analysis of probucol in plasma. J. Chromatogr. 380:401-406.

6. Blantz, R. C., and B. J. Tucker. 1978. Measurements of glomerular dynamics. In Methods in Pharmacology. Renal Pharmacology. Vol. IV. M. Martinez-Maldonado, editor. Plenum Publishing Corp. New York. 141-163.

7. Weiss, S. J., and P. A. Ward. 1982. Immune complex-induced generation of oxygen metabolites by human neutrophils. J. Immunol. 129:309-319.

8. Fantone, J. C., and P. A. Ward. 1982. Role of oxygen derived free radicals and metabolites in leukocyte dependent inflammatory reactions. Am. J. Pathol. 107:395-418.

9. Agner, K. 1941. Verdoperoxidase. A ferment isolated from leukocytes. Acta Physiol. Scand. 2(Suppl. 8):1-62.

10. Andrews, P. C., and N. I. Krinsky. 1982. Quantitative determination of myeloperoxidase using tetramethylbenzidine as substrate. Anal. Biochem. 127:346-350.

11. Ohkawa, H., N. Ohishi, and K. Yagi. 1979. Assay for lipid peroxides in animal tissues by thiobarbituric acid reaction. Anal. Biochem. 95:351-358.

12. Lowry, O. M., N. J. Rosebrough, A. L. Farr, and R. J. Randall. 1951. Protein measurement with the Folin phenol reagent. J. Biol. Chem. 193:265-275.

13. Jackson, N. M., M. C. Holden, M. E. Convery, M. D. White, and H. D. Humes. 1986. Mechanism of tert-butyl hydroperoxide (TBH)-induced injury to renal proximal tubule segments (PTS) in vitro. Clin. Res. 34:698a. (Abstr.)

14. Rehan, A., K. J. Johnson, R. G. Kunkel, and R. C. Wiggins. 1985. Role of oxygen radicals in phorbol myristate acetate-induced glomerular injury. Kidney Int. 27:503-511.

15. Aust, S. D. 1985. Lipid peroxidation. In CRC Handbook of Methods for Oxygen Radical Research. R. A. Greenwald, editor. CRC Press, Boca Raton. 203-207.

16. Donohoe, J. F., M. A. Venkatachalam, D. B. Bernard, and N. G. Levinsky. 1978. Tubular leakage and obstruction after renal ischemia: Structural-functional correlations. Kidney Int. 13:208-222.

17. Barnes, J. L., R. W. Osgood, H. J. Reineck, and J. H. Stein. 1981. Glomerular alterations in an ischemic model of acute renal failure. Lab. Invest. 45:378-386.

18. Tanner, G. A., and M. Steinhausen. 1976. Tubular obstruction in ischemia-induced acute renal failure in the rat. Kidney Int. 10:S65S73.

19. Finn, W. F. 1981. Nephron heterogeneity in polyuric acute renal failure. J. Lab. Clin. Med. 98:21-29.

20. Conger, J. D., J. B. Robinette, and S. P. Kelleher. 1984. Nephron heterogeneity in ischemic acute renal failure. Kidney Int. 26:422-429.

21. Linas, S. L., D. Whittenburg, E. Berger, and J. E. Repine. 1987. Toxic $\mathrm{O}_{2}$ metabolites from neutrophils (PMN) accentuate renal ischemia-reperfusion injury in the isolated perfused rat kidney. Kidney Int. 31:369. (Abstr.)

22. Brezis, M., S. Rosen, P. Silva, and F. H. Epstein. 1984. Transport activity modifies thick ascending limb damage in isolated perfused kidney. Kidney Int. 25:65-72. 